\title{
Heartbeat: bone densitometry for atherosclerotic risk stratification
}

Atherosclerotic vascular calcification and bone mineral density (BMD) appear to mirror each other-less bone calcification goes hand-in-hand with excess vascular calcification-with plausible biological mechanisms to explain this apparent paradox. But whether the vascular-bone calcification relationship is an independent association or is simply related to the ageing process and shared risk factors has been unclear. In this issue of Heart, Park and colleagues ${ }^{1}$ investigated the association between BMD and atherosclerotic cardiovascular disease (ASCVD) events in a cohort of 12681 women aged 50-80 years of age with a median of 9.2 years follow-up after BMD measurement. The primary endpoint of ASCVD death, non-fatal myocardial infarction or ischaemic stroke occurred in $3.7 \%$ of patients. As hypothesised, a lower BMD was associated with a higher risk for ASCVD events (adjusted HR 1.38, $\mathrm{p}<0.001$ per 1 SD decrease in BMD measured at the hip). In addition, a clinical diagnosis of osteoporosis was independently associated with a higher risk of ASCVD events even after adjustment for other risk factors (adjusted HR: 1.79, p<0.001). The authors suggest that evaluation of BMD may add incremental value for ASCVD risk stratification in women (figure 1).

In an editorial, Canoy and Rahimi ${ }^{2}$ remind us that 'The cross-talk between bones and atherosclerosis is an interesting area of research, yet it is hardly novel. In a lecture given in 1858 , Dr Rudolf Virchow referred to atherosclerotic lesions as 'ossification'. Perhaps it is high time to establish how bone health affects vasculature and understand the underlying pathophysiology that links osteoporotic and atherosclerotic conditions. In doing so, we might just discover new ways to improve the treatment of, and care for, the hearts and minds of women, as well as of men.'

Division of Cardiology, University of Washington, Seattle, Washington, USA

Correspondence to Professor Catherine M Otto, Division of Cardiology, University of Washington, Seattle, WA 98195, USA; cmotto@uw.edu

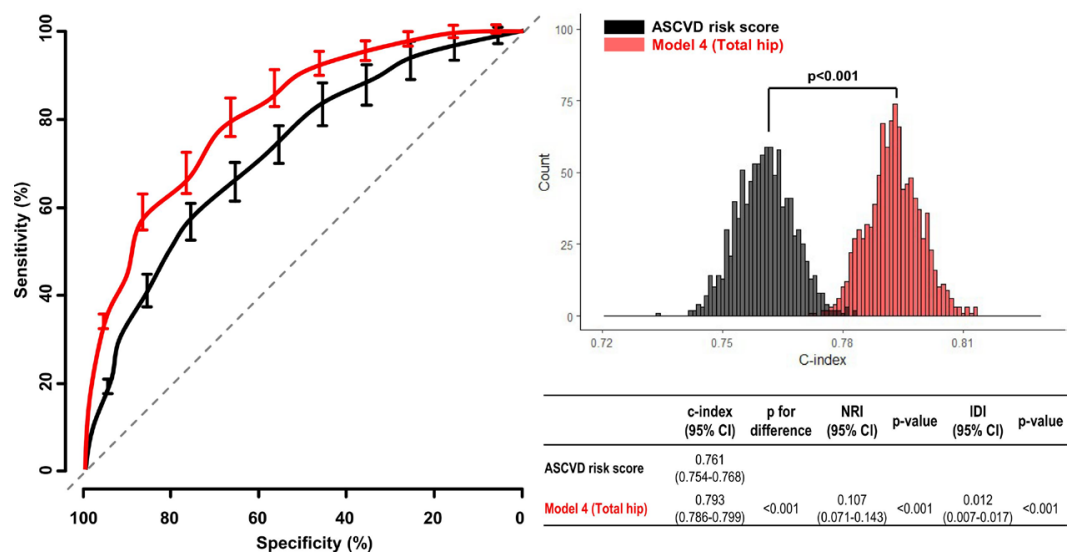

Figure 1 Comparison of prediction performance for ASCVD events between the BMD model and ASCVD risk score. The addition of a clinical diagnosis of osteopenia or osteoporosis by total hip BMD to age and other clinical risk factors demonstrates a significant improvement in model performance for predicting ASCVD events over that of the ASCVD risk score. ASCVD, atherosclerotic cardiovascular disease; BMD, bone mineral density; IDI, integrated discrimination improvement; NRI, Net Reclassification Index.

Another interesting paper in this issue of Heart is the study by Nakashima and colleagues $^{3}$ who used machine learning to predict daily out-of-hospital cardiac arrest (OHCA) incidence based on meteorological and chronological data. Using data from over 660000 OHCA cases of cardiac origin, integrated with detailed chronological and meteorological data, the machine learning model accurately predicted events as shown in figure 2. The strongest associations with OHCA incidents were days of the week (Sunday or Monday), holidays,

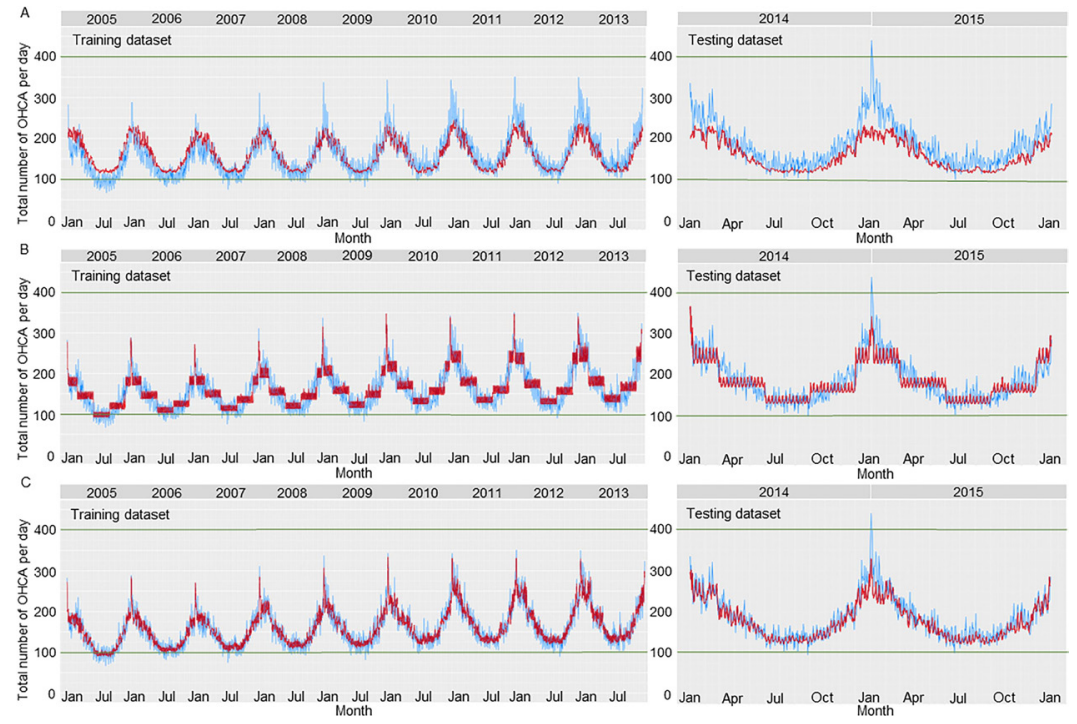

Figure 2 Observed versus predicted incidence of OHCA. The blue dots indicate the observed total number of OHCAs per day in Japan. The red dots indicate the predicted number based on the following predictive models: (A) ML model with comprehensive meteorological variables, (B) $M L$ model with chronological variables and (C) ML model with combined meteorological and chronological variables. ML, machine learning; OHCA, out-of-hospital cardiac arrest. 

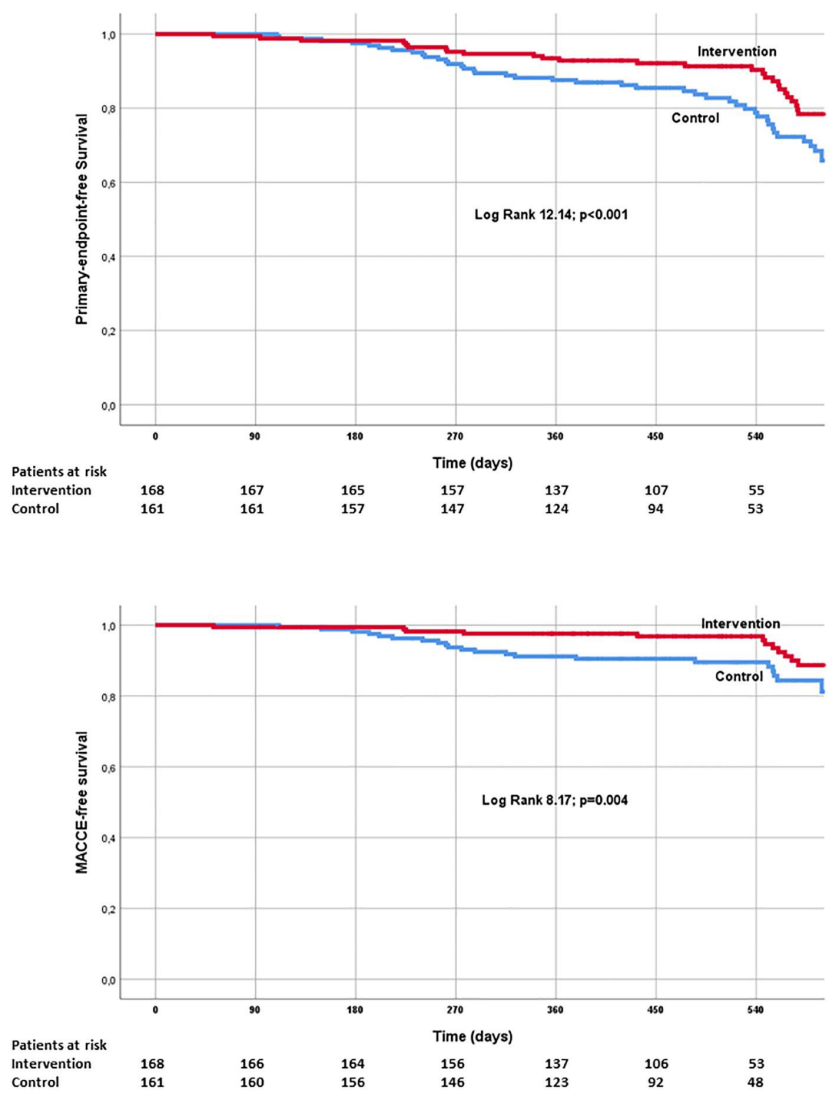

Figure 3 Kaplan-Meier curves of primary end point event-free survival (top panel) and major adverse cardiac and cerebrovascular event-free survival (bottom panel) in the two study arms.

winter, low ambient temperatures and large interday or intraday temperature differences.

The provocative editorial by Gaieski ${ }^{4}$ discusses the strengths and limitations of the study and suggests that: 'listening to the science is more important today than ever before and the intriguing data supporting an increase in mean daily

temperatures in Hokkaido (Japan's northern most prefecture) suggest the potential for global warming to cause increased incidence of cardiovascular emergencies and adds another personal, pathophysiological reason to take the climate crisis seriously. If next weeks' weather forecast is for cold and cloudy weather with an increased chance of

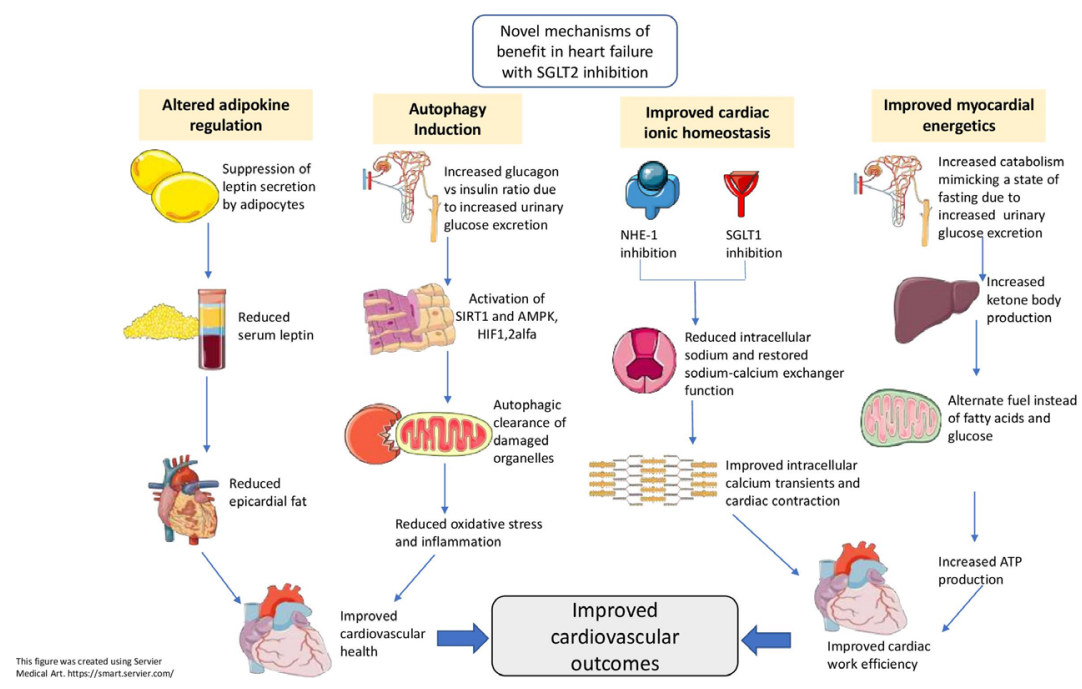

○ 2020 S Joshi

Figure 4 Schematic diagram showing proposed novel mechanisms of action of SGLT2 inhibitors in heart failure. AMPK, adenosine monophosphate-activated protein kinase; HIF, hypoxia-inducible factor; NHE, sodium-hydrogen exchanger; SGLT, sodium-glucose co-transporter; SIRT, sirtuin. cardiac arrest, citizens can be prepared, taking precautionary measures, and health systems can be staffed appropriately, ready for increased resource utilisation.'

Most physicians think that patient education is a key factor in reducing adverse cardiovascular events and in improving patient's quality of life. Yet there is sparse objective evidence for an effect of patient education on ASCVD outcomes. In this issue of Heart, Giannopoulos and colleagues ${ }^{5}$ prospectively randomised 329 patients (mostly men) after myocardial infarction to usual treatment alone versus usual treatment plus an 8-week long educational programme. The educational programme, presented by non-medical personnel, consisted of 10 hours of lessons on risk factor management, lifestyle and medical therapy. At a mean follow-up of 17 months, the primary endpoint of all-cause death, myocardial infarction, cerebrovascular event or unscheduled cardiovascular hospitalisation occurred in $20.8 \%$ of the treatment group versus $36.6 \%$ of the usual care group (OR 0.46, 95\% CI 0.28 to 0.74 ; $\mathrm{p}=0.002$ ) (figure 3). The intervention group also had greater reductions in serum low-density lipoprotein cholesterol, systolic blood pressure and body mass index, compared with the control group.

The effects of the educational programme on clinical outcomes in the study by Giannopoulos ${ }^{5}$ are impressive but, as Taylor points out in his editorial, ${ }^{6}$ for this approach to be effectively implemented in other institutions additional reporting elements are needed including 'the intervention underlying theoretical basis (how will the intervention cause change?), method of intervention development (eg, were patients, clinicians and other key stakeholders involved in the intervention design?), fidelity of delivery (eg, what checks were in place in the trial to check that the intervention was delivered as planned?) and the costs and resources (eg, staffing) to deliver the intervention.'

The Education in Heart article in this issue $^{7}$ addresses the underlying genetics, diagnosis, risk stratification and treatment options for the major ion channelopathies including long QT syndrome, Brugada syndrome and catecholaminergic polymorphic ventricular tachycardia.

Readers will also want to look at the excellent review article $^{8}$ on the novel mechanisms of action of sodium-glucose 
co-transporter 2 inhibitor therapy in patients with heart failure (figure 4).

Our Cardiology-in-Focus article ${ }^{9}$ in this issue summarises the unique cardiovascular health concerns in sexual and gender minority (SGM) persons, along with approaches to primary and secondary ASCVD prevention. As the authors conclude: 'SGM individuals represent a unique subset of patients that have increased CVD risk related to behavioural, psychosocial and physiological factors. Even though there is a growing body of research documenting cardiovascular health disparities among SGM populations, efforts to specifically address these concerns can be improved, including clinician participation in data collection, awareness of unique factors contributing to disparate health outcomes, revising educational content and populationbased improvements in methodological approaches.'
Funding The authors have not declared a specific grant for this research from any funding agency in the public, commercial or not-for-profit sectors.

Competing interests None declared.

Patient and public involvement Patients and/or the public were not involved in the design, or conduct, or reporting, or dissemination plans of this research.

Patient consent for publication Not required.

Provenance and peer review Commissioned; internally peer reviewed.

(c) Author(s) (or their employer(s)) 2021. No commercial re-use. See rights and permissions. Published by BMJ.

\section{(D) Check for updates}

To cite Otto CM. Heart 2021;107:1021-1023.

Heart 2021;107:1021-1023.

doi:10.1136/heartjnl-2021-319755

ORCID iD

Catherine M Otto http://orcid.org/0000-0002-05279392

\section{REFERENCES}

1 Park J, Yoon YE, Kim KM, et al. Prognostic value of lower bone mineral density in predicting adverse cardiovascular disease in Asian women. Heart 2021;107:1040-6.

2 Canoy D, Rahimi K. A way to a woman's heart might be through her bones. Heart 2021;107:1024-5.

3 Nakashima T, Ogata S, Noguchi T. Machine learning model for predicting out-of-hospital cardiac arrests using Meteorological and chronological dataMachine learning model for predicting out-of-hospital cardiac arrests using Meteorological and chronological data. Heart 2021:107:1084-91.

4 Gaieski DF. Next week's weather forecast: cloudy, cold, with a chance of cardiac arrest. Heart 2021:107:1030-1.

5 Giannopoulos G, Karageorgiou S, Vrachatis D, et al. A stand-alone structured educational programme after myocardial infarction: a randomised study. Heart 2021:107:1047-53.

6 Taylor R. Postmyocardial infarction education is effective but not enough on its own. Heart 2021;107:1026-7.

7 Mellor GJ, Behr ER. Cardiac channelopathies: diagnosis and contemporary management. Heart 2021;107:1092-9.

8 Joshi SS, Singh T, Newby DE. Sodium-Glucose cotransporter 2 inhibitor therapy: mechanisms of action in heart failureSodium-Glucose co-transporter 2 inhibitor therapy: mechanisms of action in heart failure. Heart 2021;107:1032-8.

9 Streed C, Caceres B, Mukherjee M. Preventing cardiovascular disease among sexual and gender minority persons. Heart 2021;107:1100-1. 